\title{
ANALYTICAL APPROXIMATION OF TIME-FRACTIONAL TELEGRAPH EQUATION WITH RIESZ SPACE-FRACTIONAL DERIVATIVE
}

\author{
SAFiYeh Mohammadian, Yaghoub Mahmoudi And FARHAD Dastmalchi \\ SAEI
}

Abstract. In this study, fractional reduced differential transform method (FRDTM) is developed to derive a semi- analytical solution of fractional partial differential equations which involves Riesz space fractional derivatives. We focus primarily on implementing the novel algorithm of FRDTM to Riesz space -fractional telegraph equation while the telegraph equation has fractional order. Some theorems with their proofs which are used for calculating differential transform of Riesz derivative operator are presented, as well as the convergence condition and the error bound of the proposed method are established. To illustrate the reliability and capability of the method, some examples are provided. The results reveal that the algorithm is very effective and uncomplicated.

Mathematics subject classification (2010): 65Z05, 35Q60, 35Q99.

Keywords and phrases: Telegraph equation, Riesz space fractional derivative, fractional derivatives, fractional reduced differential transform method, reduced differential transform method.

\section{REFERENCES}

[1] Y. ZhENG, Z.ZHAO, The time discontinuous space-time finite element method for fractional diffusionwave equation, Appl. Numer. Math., 150, (2020), 105-116.

[2] Z. ZHAU, C. LI, Fractional difference finite element approximations for time-space fractional telegraph equation, Appl. Math. Comput., 219, 6 (2012), 2975-2988.

[3] Q. YANG, F. LIU, I. TURNER, Numerical methods for fractional partial differential equations with Riesz space fractional derivatives, Appl. Math. Model., 34, (2010), 200-218.

[4] A. H. Bhrawy, M. ZAKy, J. A. Tenreiro Machado, Numerical solution of the two-sided-time fractional telegraph equation via Chebyshev Tau approximation, J. Optimal. Theory. Appl., 174, 1 (2017), 321-341.

[5] V. R. Hossieni, W. Chen, Z. AvazZadeh, Numerical solution of fractional telegraph equation by using radial basis function, Eng. Anal. Bound. Elem., 38, (2014), 31-39.

[6] Y. ZHENG, Z. ZHAO, The discontinuous Galerkin finite element method for fractional cable equation, Appl. Numer. Math., 115, (2017), 32-41.

[7] S.Chen, X. JiAng, F. LiU, I. TURnER, High order unconditionally stable difference schemes for the Riesz space-fractional telegraph equation, J. Comput. Appl. Math., 278, (2015), 119-129.

[8] Y. ZHANG, H. DiNG, Improved matrix transform method for the Riesz space fractional reaction dispersion equation, J. Comput. Appl. Math., 260, (2014), 266-280.

[9] Z. ZHAO, Y. ZHENG, P.GuO, A Galerkin finite element method for a class of time -space fractional differential equation with nonsmooth data, J. Sci. Comput., 70, (2017), 386-406.

[10] J. K. ZHOU, Differential Transformation and its Application for Electrical Circuits, Huazhong University Press, Wuhan, China, 1986 (in Chinese).

[11] C. K. Chen, S. H. Ho, Solving partial differential equations by two dimensional differential transform, Appl. Math. Comput., 106, (1999), 171-179.

[12] Z. Odibat, S. Momani, V. S. ERTURK, Generalized differential transform method: Application to differential equations of fractional order, Appl. Math. Comput., 197, (2008), 467-477. 
[13] Z. Odibat, S. Momani, A generalized differential transform method for linear partial differential equations of fractional order, Appl. Math. Lett., 21, (2008), 194-199.

[14] B. SOLTANIZADEH, differential transform method for solving one-space-dimensional telegraph equation, Comput. Appl. Math. 30, 3 (2011), 639-653.

[15] V. K. Sirvastava, M. K. Awasthi, R. K. Chaurasia, Reduced differential transform method to solve two and three dimensional second order hyperbolic telegraph equations, J. King Saud Univ., Eng. Sci., 29, 2(2017), 166-171.

[16] M. Garg, P. Manogar, S. L. Kalla, Generalized differential transform method to space- time fractional telegraph equation, Int. J. Differ. Equations., Article ID 548982, (2011), 9 pages.

[17] Y. Keskin, G. OTURANÇ, Reduced differential transform method for partial differential equations, Int. J. Nonlin. Sci. Num., 10, 6 (2009), 741-749.

[18] M. ARSHAD, D. LU, J. WANG, (N+1)-dimensional fractional reduced differential transform method for fractional order partial differential equations, Commun. Nonlinear. Sci., 48, (2017), 509-519.

[19] V. K. SRIVAstaVA, M. K. Awasthi, S. Kumar, Analytical approximations of two and three dimensional time-fractional telegraph equation by reduced differential transform method, Egypt. J. Basic. Appl. Sci., 1, 1 (2014), 60-66.

[20] S. AbUaSAD, I. S. HASHIM, A. KARIM, Modified fractional reduced differential transform method for the solution of multi term time-fractional diffusion equation, Adv. Math. Phys., Article ID 5703916, (2019), 14 pages.

[21] D. Lu, J. Wang, M. Arshad, A. Abdullah, A. Ali, Fractional reduced differential transform method for space-time fractional order heat-like and wave-like partial differential equations, J. Adv. Phys., 7, (2018), 1-10.

[22] I. Podlubny, Fractional Differential Equations, Academic Press, SanDiego, 1999.

[23] K. S. Miller, B. Ross, An Introduction to the Fractional Calculus and Fractional Differential Equations, John Wiley \& Sons Inc., New York, 1993.

[24] C. LI, M. CAI, Theory and Numerical Approximations of Fractional Integrals and Derivatives, SIAM, Philadelphia, 2019.

[25] C. Li, F. ZENG, Numerical Methods for Fractional Calculus, Chapman and Hall/CRC, Boca Raton, USA, 2015. 